\title{
Simple Models of Human Locomotion
}

\author{
R. MCNEILL ALEXANDER* \\ Department of Biology, University of Leeds, Leeds LS2 9JT, UK
}

(Received 1 October 1996)

\begin{abstract}
The human body is a complex structure, but our understanding of its movements is greatly enhanced by extremely simple mathematical models. A model of walking shows why we have to break into a run at speeds above 3 metres per second, and why the critical speed is lower for children and on the moon. A model of running jumps shows why long jumpers run up at a fast sprinting speed, but high jumpers run up much more slowly, Finally, a model of standing jumps explains why athletes can jump higher by using a countermovement than from a static squatting position. The body is represented in these models as a small number of rigid segments connected by hinge joints, powered by muscles with realistic physiological properties.
\end{abstract}

Keywords: walking, jumping, biomechanics

\section{THE COMPLEXITY OF THE BODY}

Much of our understanding of human movement depends on mathematical modelling, but models even of the same parts of the body differ dramatically in complexity. The body itself is extremely complex with its many movable joints, and some authors have chosen to model as much as possible of this complexity. In a classic paper on standing, Seireg and Arvikar (1973) modelled each leg as an assembly of three rigid segments (foot, lower leg and thigh) connected together by joints allowing a total of six degrees of freedom of relative movement (two at the ankle, one at the knee and three at the hip). What I have described so far is relatively simple, and indeed is simplified (for example, it allows no flexibility in the toes) but it was powered by no fewer than 29 muscles. Here again we have simplification (real legs have many more muscles than that) but with more muscles than degrees of freedom it was impossible to calculate the forces that would act in individual muscles without making more-or-less arbitrary assumptions. That did not by any means destroy the value of the model, which was designed to discover what patterns of muscle activity were predicted by different assumptions. The predicted patterns were compared to actual ones, recorded by using electrodes to detect the electrical activity that accompanies force production in muscles.

In another excellent paper, Yeadon (1990) set out to explain how trampolinists execute twisting somersaults. He was not concerned with individual muscles, but only with the effects of movements of body

\footnotetext{
${ }^{*}$ Tel: +441132332911 Fax: +441132332835
} 
segments, relative to each other, on the movement of the body as a whole. He simplified body structure, treating the hands and feet as rigid extensions of the forearms and lower legs, and putting just one joint in the back instead of a long row of movably-jointed vertebrae. That reduced the number of segments to eleven, but he still had to cope with a formidable array of equations. Using this model he was able to show, for example, how a trampolinist in midair could convert a straight somersault to a twisting one simply by raising one arm, and he successfully predicted the degree of twist.

Those examples are towards the complex end of the range. My next example shows at the other extreme how a model that may seem ludicrously simple may nevertheless contribute to our understanding.

\section{WALKING}

In walking, each knee is kept straight while the foot of the same leg is on the ground, so our bodies bob up and down as we walk. The centre of mass of the whole body is a very few centimetres above the hip joints. These observations suggested the model shown in Figure 1 which has straight, massless legs of constant length and a rigid trunk with its centre of mass at the hip (Alexander, 1976). As it walks, the centre of mass advances along a series of arcs of circles. We will use the model to ask why we walk only at low speeds, and break into a run to go fast. In running, our knees bend appreciably while the foot

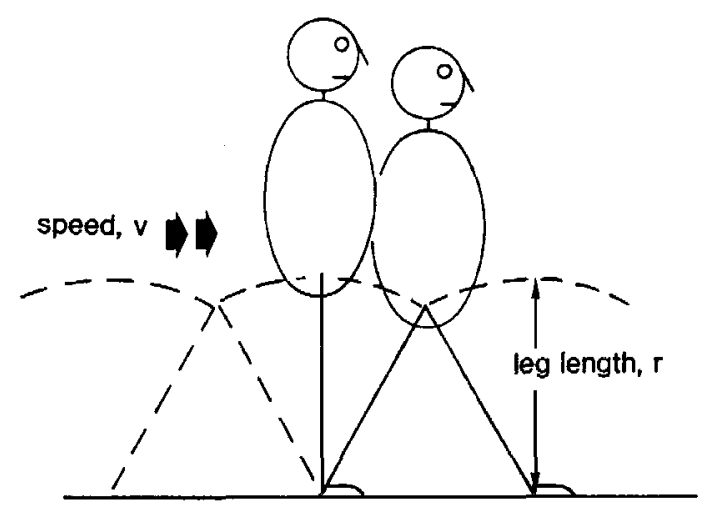

FIGURE 1 A model of walking. is on the ground. Also, in walking each foot is on the ground for more than half the time, so there are stages of the stride when both are on the ground; but in running each foot is on the ground for less than half the time, so at times both of them are off. Adult people prefer to change from walking to running when their speed reaches $2 \mathrm{~m} \mathrm{~s}^{-1}$ and cannot walk faster than $2.5-3.0 \mathrm{~m} \mathrm{~s}^{-1}$ except by using a special technique, the racing walk.

At the stage when the supporting leg is vertical in Figure 1, the centre of mass is moving with speed $v$ along an arc of a circle of radius $r$. Therefore, it has a downward acceleration $v^{2} / r$. This cannot exceed the gravitational acceleration $\mathrm{g}$.

$$
\begin{aligned}
v^{2} / r & \leq \mathrm{g} \\
v & \leq(\mathrm{g} r)^{0.5}
\end{aligned}
$$

An adult's leg length is about $0.9 \mathrm{~m}$, and $\mathrm{g}$ is about $10 \mathrm{~m} \mathrm{~s}^{-2}$ so this inequality tells us it is impossible to walk faster than $3 \mathrm{~m} \mathrm{~s}^{-1}$. It also seems to explain why small children often have to run to keep pace with a walking adult, giving a speed limit of $2 \mathrm{~m} \mathrm{~s}^{-1}$ for a two-year old with 0.4-m legs. Further, it suggests a possible reason why walking was not the preferred gait of astronauts on the moon, where $\mathrm{g}$ is only $1.6 \mathrm{~m} \mathrm{~s}^{-2}$. In the racing walk, the pelvis tilts when the supporting leg is vertical, reducing the vertical excursion of the centre of mass and making higher speeds possible: the world record speeds for the $10 \mathrm{~km}$ walking race are $4.4 \mathrm{~m} \mathrm{~s}^{-1}$ (men) and $4.0 \mathrm{~m} \mathrm{~s}^{-1}$ (women).

\section{HIGH JUMPING}

It is seldom possible to build a useful model as simple as that one. Often, we need to incorporate muscles with realistic properties.

Muscles can be thought of as actuators in series with springs (Figure 2(a)). In most cases, the elastic compliance resides mainly in the tendons which connect the muscle to the skeleton. The force in the spring is a function of its length $s$ (Figure 2(b)), but the force that the muscle actuator exerts, when fully activated, is a function of its rate of change of length, $\mathrm{d} a / \mathrm{d} t$ (Figure 2(c)). The faster the actuator 


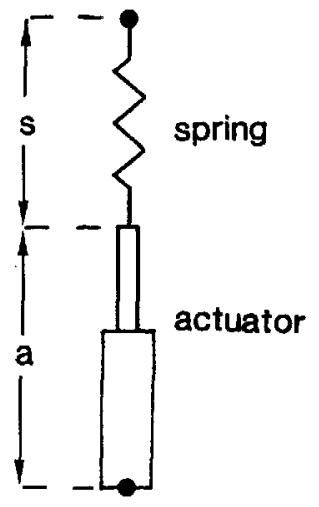

a

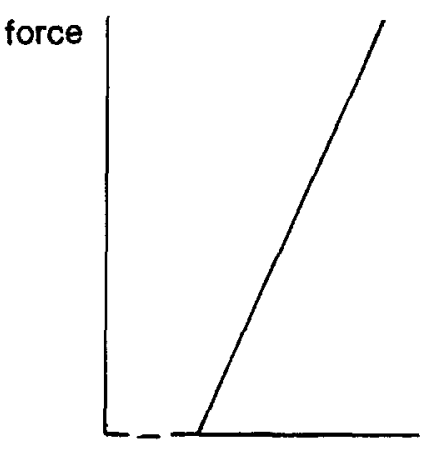

$\mathbf{s}$

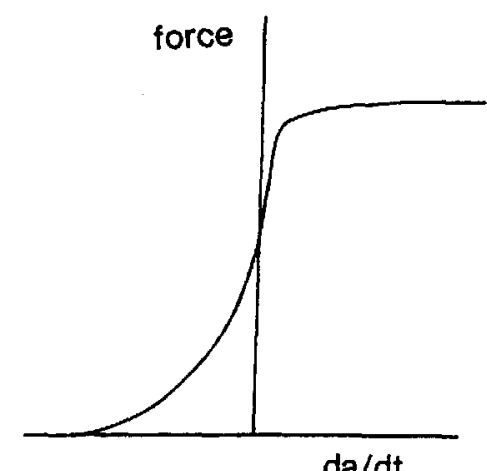

C

FIGURE 2 (a) A diagram of a muscle as an actuator in series with a spring. (b,c) Schematic graphs showing (b) that the force in the spring is a function of its length and (c) that the force that the actuator can develop is a function of its rate of change of length.

is shortening, the less force it exerts, but it can exert increased force when being forcibly stretched. The relationships shown schematically by the graphs in Figure 2 have been shown to be similar for a wide variety of muscles, and can be represented by empirical equations.

A muscle model of the kind represented in Figurc 2 has been used in a study of human jumping. (Alexander, 1990). The aim was to explain a striking difference between high jumpers and long jumpers. Good long jumpers run up for take-off at high sprinting speeds, of $10 \mathrm{~m} \mathrm{~s}^{-1}$ or more. In contrast, good high jumpers run up slowly, at only $7 \mathrm{~m} \mathrm{~s}^{-1}$. A high jumper who ran up at $10 \mathrm{~m} \mathrm{~s}^{-1}$ would have twice as much kinetic energy available for conversion to gravitational potential energy, and might be expected to rise higher. Why do high jumpers run up so slowly?

The model (Figure 3(a)) is similar in some respects to the model of walking. The trunk is rigid and the masses of the legs are ignored. However, there is a flexible knee joint, powered by an extensor muscle with the properties shown in Figure 2. The model runs up at speed $u$ and places its foot on the ground with the knee (almost) straight at an angle $\theta$ to the horizontal. The muscle is fully activated and remains so until the foot leaves the ground. The knee bends and extends again (as happens when a real athlete takes off), and the athlete is thrown into the air. This course of events is followed by numerical integration on a personal computer which goes on to determine the trajectory of the centre of mass after take-off.

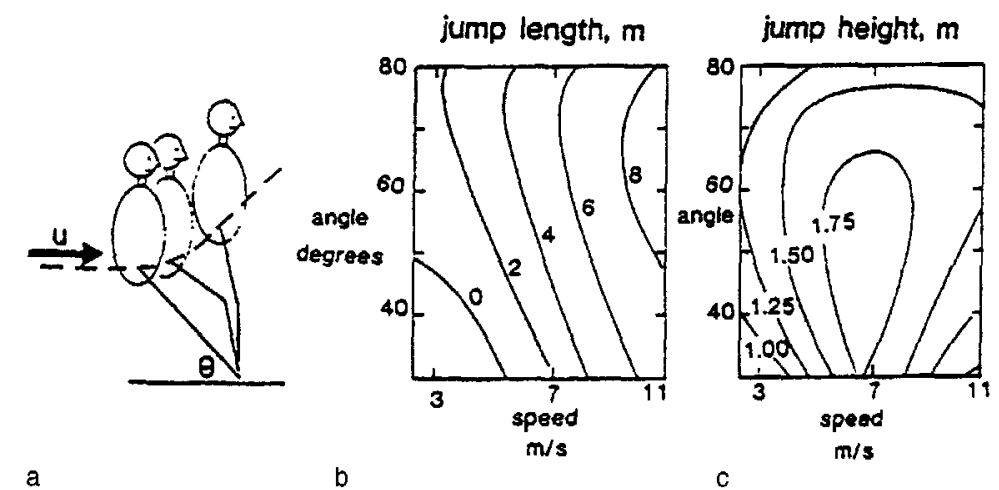

FIGURE 3 (a) A model of high or long jumping. (b) The lengths and (c) the heights in metres of jumps, as predicted by the model for different run-up speeds $u$ and initial leg angles, $\theta$. From Alexander (1995b). 
The contortions performed by high jumpers in the air enable them to pass their centres of mass either very close above the bar or even under it, so the program estimates the height of the jump as the greatest height attained by the centre of mass. The length of the jump is estimated reasonably realistically as the horizontal distance the centre of mass would travel before hitting the ground if it were an isolated particle.

Calculations were performed for a range of speeds $u$, from a slow jog at $2.5 \mathrm{~m} \mathrm{~s}^{-1}$ to a recordbreaking sprint at $11 \mathrm{~m} \mathrm{~s}^{-1}$, and for a range of initial leg angles $\theta$. The results are shown as contours in Figure $3(b, c)$. Figure $3 b$ shows that the longest jumps are obtained by running up as fast as possible and setting down the leg at about $65^{\circ}$ (which is what good long jumpers do). Figure 3(c) shows that the highest jumps should be obtained by running up at about $7 \mathrm{~m} \mathrm{~s}^{-1}$ and setting down the leg at $45-50^{\circ}$ (which is what high jumpers do). These conclusions are remarkably insensitive to the values of the parameters of the relationships shown in Figure $2(b, c)$, which is fortunate because some of these parameters are not at all precisely known for human muscle.

The counterintuitive conclusion, that high jumpers should run up quite slowly, emerges clearly from the mathematics. It seems useful to try to explain it in words. What a high jumper wants to do is to exert as large as possible a vertical impulse on the ground, so as to gain the highest possible vertical component of velocity of take-off. Because the force that can be exerted on the ground is limited by the properties of the muscle, the impulse (the time integral of the force) can be large only if the foot remains on the ground for a reasonably long time. It cannot remain at one point on the ground for long if the athlete is running fast.

\section{STANDING JUMPS}

The last of the models that we will examine in this paper is another model of jumping, but this one represents standing jumps rather than running ones
(Alexander, 1995a). Humans, bushbabies, frogs, grasshoppers, fleas and many other animals jump by forcibly extending their hind legs, but there is a notable difference between the jumping techniques of humans and of insects. We have to consider three different techniques.

(i) The squat jump. Starting with the knees bent, the jumper activates the leg muscles which develop tension and extend the knees, throwing him or her into the air.

(ii) The catapult jump. Grasshoppers and fleas have catch mechanisms in their legs that can hold the knee bent even when the jumping muscles are exerting maximum force. With the catch operating, the muscles are activated. Only when their force has reached its peak is the catch released, allowing the jump to proceed.

(iii) The countermovement jump. Athletes making standing jumps start with their legs straight. They bend their knees and then immediately re-extend them, throwing themselves into the air. In one experiment it was found that basketball players could jump $6 \mathrm{~cm}$ higher by using a countermovement, than they could in a squat jump.

Figure 4(a) shows a model designed to investigate these different styles of jumping. It is highly generalized and will serve (with different parameters) to represent a human athlete or a jumping insect. Its symmetry simplifies the analysis.

As in the previous models, the trunk is rigid. Each leg has two segments, each of which is a rod of appropriate mass (a difference from the previous models whose legs were massless). The knee joints are powered by extensor muscles which have the properties illustrated in Figure 2. The course of events is again followed by numerical integration, in a computer program.

Figure 5 shows sample simulations of the three types of jump. In each case knee angle, and the moment that the muscles exert at the knee, are plotted against time. The broken horizontal line shows in each case the moment the muscle can exert 


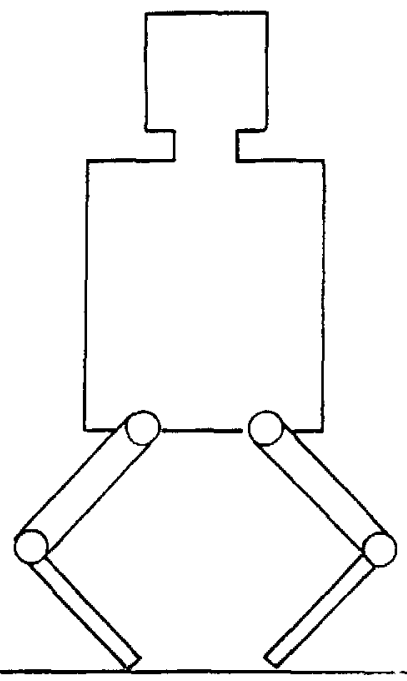

a

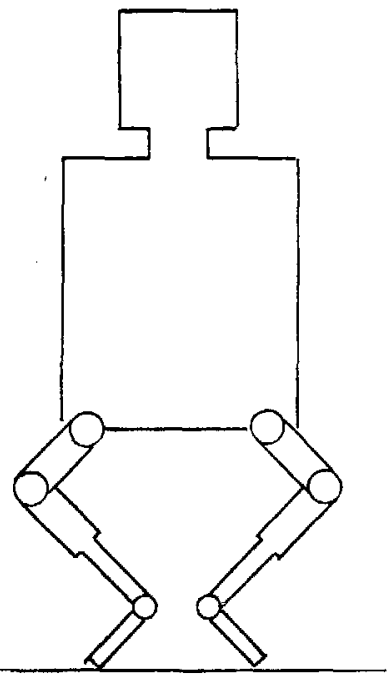

b

FIGURE 4 Two models considered in the discussion of standing jumps.

when contracting isometrically (i.e. when $\mathrm{d} a / \mathrm{d} t=$ 0 ; see Figure 2).

The squat jump (Figure 5(a)) starts with the knees bent and the muscles inactive. At time zero, the muscles are active and start contracting. Initially, the moment is low and the muscle actuators shorten rapidly. This stretches the tendon springs and the moment builds up. The knees extend at an accelerating rate until the feet leave the ground. The moment never reaches the isometric value because, throughout takeoff, $\mathrm{d} a / \mathrm{d} t<0$.

The catapult jump (Figure 5(b)) starts with the catch in place and the muscles exerting their isometric moment. At time zero the catch is released, the muscles shorten at an increasing rate and the force they can exert falls. As it falls, the elastic recoil

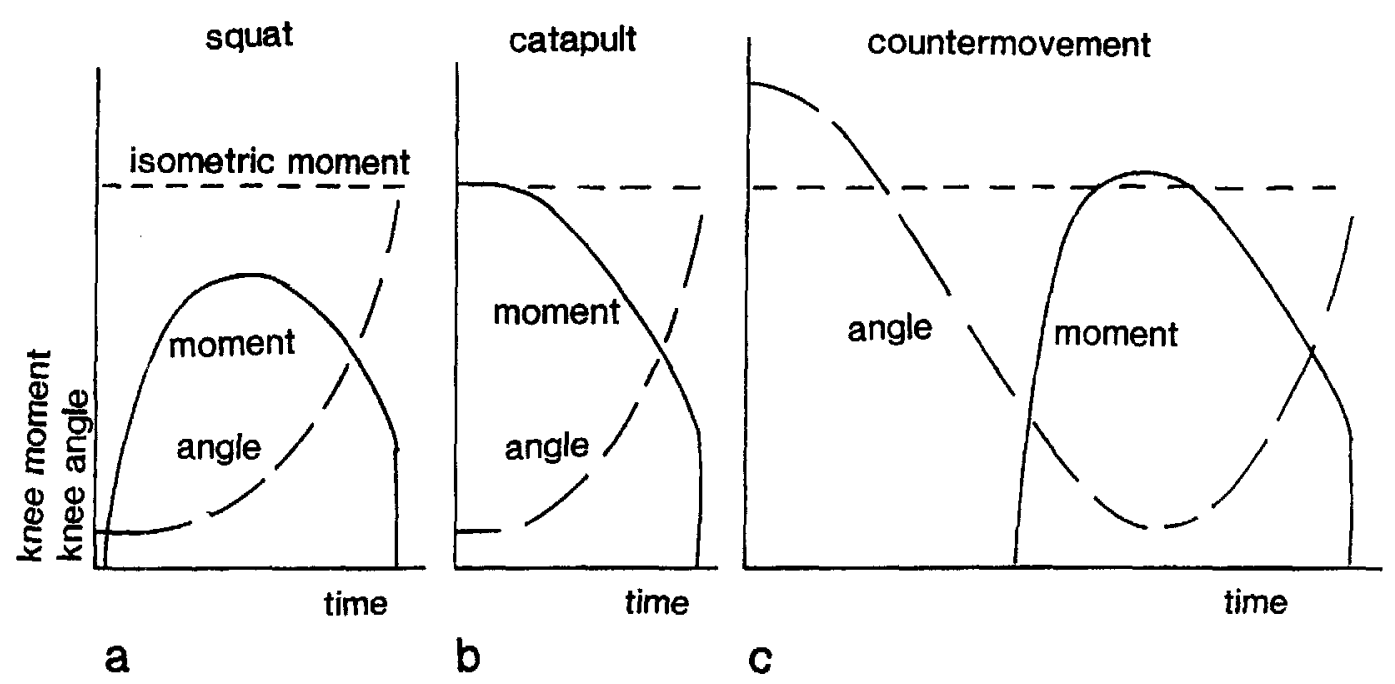

FIGURE 5 Graphs showing the time courses of knee angle and of the moment exerted by the knee muscles, in simulated jumps by. the models shown in Figure 4(a). (a) Represents a squat jump; (b) a catapult jump; and (c) a countermovement jump. 
of the tendon springs releases energy that helps the jump. Observations on insects show that very little shortening of the muscles occurs as the legs extend: the insect is catapulted into the air almost entirely by the elastic recoil of the initially-stretched tendon springs.

The countermovement jump (Figure 5(c)) starts with the knees straight, but they bend as the trunk falls under gravity. At the appropriate instant, the knee muscles are activated and build up tension, halting the trunk's fall and projecting it upwards again. In the fall, the muscle actuators are forcibly stretched $(\mathrm{d} a / \mathrm{d} t$ is positive, Figure $2(\mathrm{a}))$ and the knee moment rises above the isometric value. It remains above isometric in the very early stages of knee extension when although the muscle as a whole is shortening $(\mathrm{d} a / \mathrm{d} t+\mathrm{d} s / \mathrm{d} t)$ is negative), the force is still high enough to stretch the actuators $(\mathrm{d} a / \mathrm{d} t$ is positive).

Humans making standing jumps exert peak forces on the ground at take-off of 2-2.5 times body weight. Insects exert peak forces ranging from about 20 times body weight for a locust to over 100 times body weight for a flea. The differences are largely explicable by the differences in body size: for geometrically similar animals, weight is proportional to the cube of linear dimensions but peak forces are expected to be proportional to muscle crosssectional area, that is to the square of linear dimensions. Simulations have been performed for jumpers of different sizes, giving the model's muscles appropriate strengths.

The simulations show that if the muscles have no elastic compliance, the three styles of jumping are equally effective. This is because, without compliance, the knee moment in a squat jump rises instantaneously to the isometric value when the muscle is activated; and the moment in a countermovement jump falls to the isometric value when knee bending is halted. Therefore the moments for the three styles of jump are the same at the start of (and throughout) knee extension. However, when the muscle is given realistic compliance (by including the spring shown in Figure 2(a)) the three techniques give jumps of different heights. For a human-sized jumper, countermovement and catapult jumps are about equally high, and clearly better than squat jumps. For insect-sized jumpers, however, catapult jumps are much better than either squat or countermovement jumps. This is consistent with the observation that humans perform countermovement jumps whereas insects use a catapult mechanism. The difference between humans and insects can be explained by the observation that the gravitational potential energy lost in a human countermovement can be stored as elastic strain energy in the tendons and made available for the jump by elastic recoil. It represents a substantial fraction of the potential energy gained in the jump. The potential energy that a flea would lose in a countermovement, however, would be a trivial fraction of the energy needed for the jump. (Fleas and other insects jump to heights that are many times the length of their legs.)

We have noted that the model for standing jumps (unlike the one for running jumps) has legs of finite mass. This refinement was not necessary for the simulations described so far, but I wanted to explore the effects of leg mass on jumping performance. I found as expected that the smaller the fraction of body mass that was in the legs, the higher the jump.

A less intuitive result emerged when the model shown in Figure 4(b) was investigated. This has legs of the same length and mass as the model of Figure 4(a), with the mass distributed in the same way along the length of the leg. However, each leg has an additional joint - an ankle as well as a knee. The ankle and knee are constrained always to have equal angles and the two joints together are powered by the same quantity of muscle, with the same properties as powered the knee in Figure 4(a). In the simulations, model (b) jumped higher than model (a). The reason seems to be this: as the legs extend, each particle in them has to be given a horizontal component of velocity. The corresponding kinetic energy has to be supplied by muscular work, but contributes nothing to the potential energy gained in the jump. With the extra joints in the legs, the horizontal displacements of leg particles are smaller, so less kinetic energy has to be given to them. This result helps to explain why 
some specialized jumping animals have additional segments in their legs, notably the elongated pelvis of frogs. This is moveably jointed to the backbone as well as to the femur and functions effectively as an additional leg segment. The hunchbacked appearance of a frog preparing to jump is due to this joint being bent.

\section{OTHER SIMPLE MODELS}

The models described here are only a small selection from among many simple models of human movement: Alexander (1995b) gives a more extensive review. Simple models of walking have been designed to tackle many different questions. Do the legs swing forward passively like compound pendulums? Is walking an inherently stable motion? Why does the pattern of force that we exert on the ground change gradually as we walk at increasing speed, and why do we change abruptly to running (with a very different force pattern) at a particular speed, which is lower than the highest possible walking speed? Each question required a different simple model, designed to extract the features that are essential for that question from the unmanageable complexity of the human body. Similarly, different models have been devised to answer different questions about jumping, for example about the coordination of the various muscles involved and about the problem of clearing the bar (in high jumping) without touching it with any part of the body. Again, models of the same activity designed for different objectives may look very different from each other.

The models described here (and by Alexander, 1995b) may seem at first sight excessively simple, but I believe that their simplicity is their strength. The simpler the model, the clearer it is which of its features are essential to the observed effect.

\section{References}

Alexander, R. McN. (1976) Mechanics of bipedal locomotion, in P. Spencer Davies (ed.) Perspectives in Experimental Biology, (Pergamon, Oxford), 1, 493-504.

Alexander, R. McN. (1990) Optimum take-off techniques for high and long jumps. Philosophical Transactions of the Royal Society B, 329, 3-10.

Alexander, R. McN. (1995a) Leg design and jumping technique in humans, other vertebrates and insects. Philosophical Transactions of the Royal Society B, 347, 235-248.

Alexander, R. McN. (1995b) Simple models of human movement. Applied Mechanics Reviews, 48, 461-470.

Seireg, A. and Arvikar, R. J. (1973) A mathematical model for evaluation of forces in lower extremities of the musculoskeletal system. Journal of Biomechanics, 6, 313-326.

Yeadon, M. R. (1990) The simulation of aerial movement. II: A mathematical inertia model of the human body. Journal of Biomechanics, 23, 67-74. 


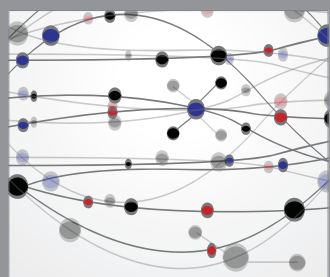

The Scientific World Journal
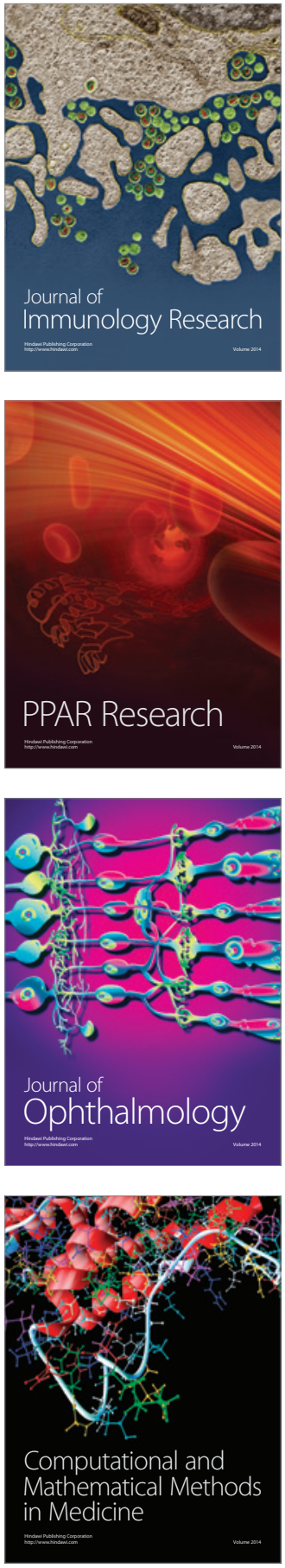

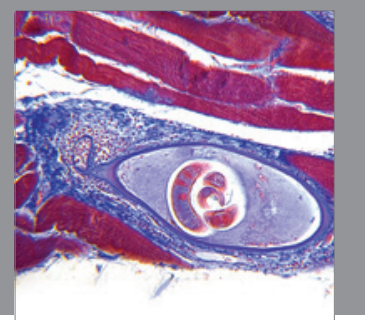

Gastroenterology

Research and Practice
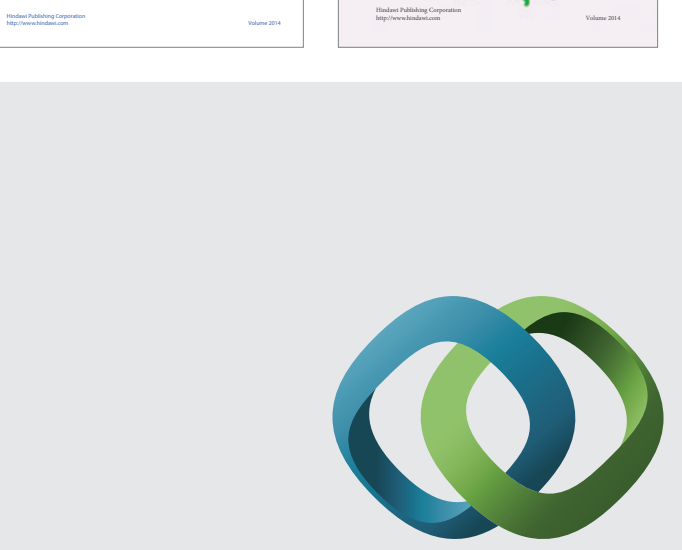

\section{Hindawi}

Submit your manuscripts at

http://www.hindawi.com
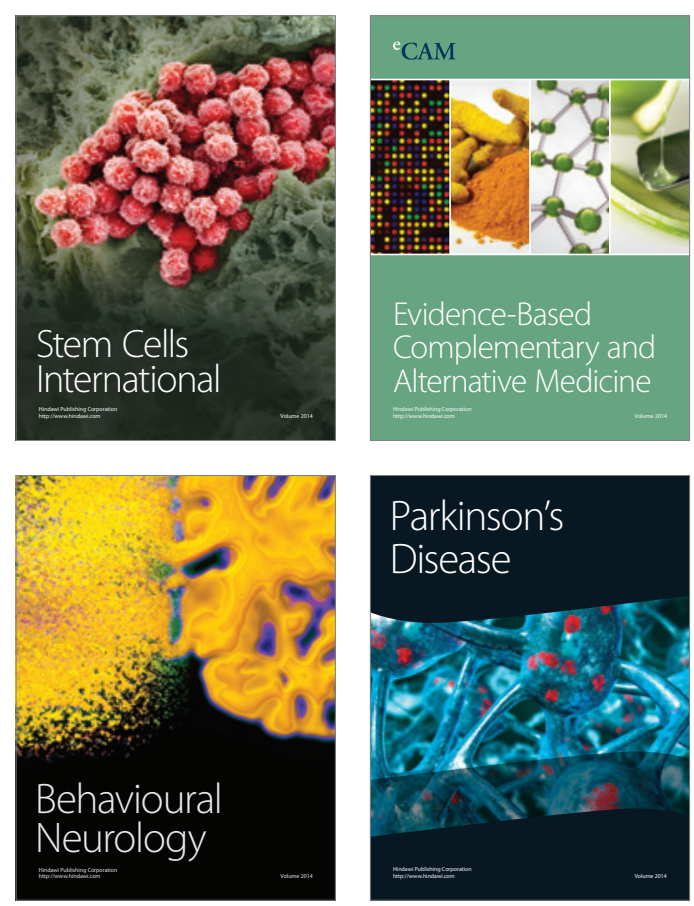

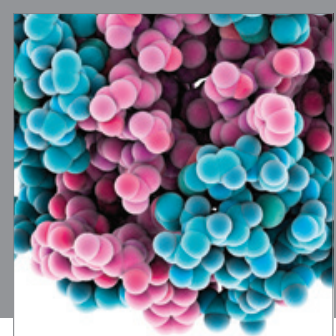

Journal of
Diabetes Research

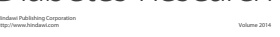

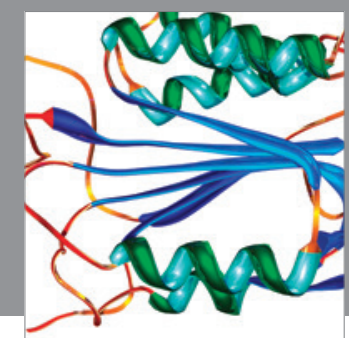

Disease Markers
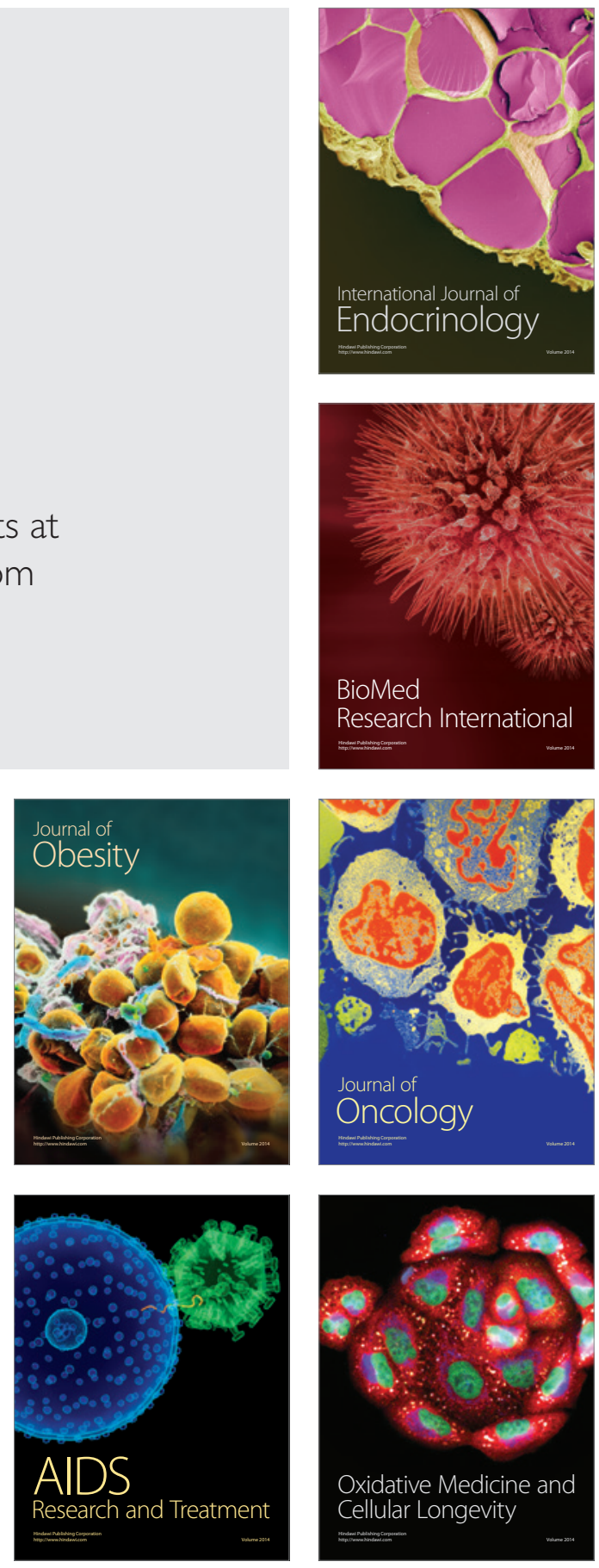